\title{
PMX-DHP Therapy for Dyspnea and Deoxygenation in Severe COVID-19 Pneumonia: A Case Series
}

This article was published in the following Dove Press journal: Infection and Drug Resistance

\section{Tsukasa Kuwana (D) \\ Kosaku Kinoshita (D) \\ Marina Hirabayashi \\ Shingo Ihara (D) \\ Nami Sawada \\ Tomokazu Mutoh (D) \\ Junko Yamaguchi (D)}

Division of Emergency and Critical Care Medicine, Department of Acute Medicine, Nihon University School of Medicine,

Tokyo, Japan
Correspondence: Kosaku Kinoshita Division of Emergency and Critical Care Medicine, Department of Acute Medicine, Nihon University School of Medicine, 30-I Oyaguchi Kamichou Itabashi-ku, Tokyo, Japan

Tel +8I-3-3972-8III

Fax +8I-3-5995-1069

Email kinoshita.kosaku@nihon-u.ac.jp
Abstract: Hypercytokinemia induced by coronavirus disease-19 (COVID-19) is associated with severe pulmonary involvement, which may lead to respiratory failure. These conditions play an important role in the worsening of clinical symptoms in patients with severe COVID19. There is no established treatment for hypercytokinemia. We report on two patients whose clinical symptoms improved after direct hemoperfusion using polymyxin B-immobilized fiber column (PMX-DHP), following the administration of the anti-inflammatory agent tocilizumab. Case A was a 70-year-old man diagnosed with COVID-19 pneumonia. Despite treatment with ciclesonide and favipiravir, supplemental oxygen was administered due to the worsening of dyspnea with tachypnea. Although tocilizumab was started on day 6 , the patient deteriorated into deoxygenation, presenting with the $\mathrm{PaO}_{2} / \mathrm{F}_{\mathrm{I}} \mathrm{O}_{2}(\mathrm{P} / \mathrm{F})$ ratio of 92 On days 8 and 10, the patient received PMX-DHP therapy. On day 11, his dyspnea improved. On day 13 , his $\mathrm{P} / \mathrm{F}$ ratio began to improve, and oxygen therapy was discontinued on day 18 . The patient recovered without requiring mechanical ventilation. Case B was a 70-year-old man diagnosed with COVID-19 pneumonia and treated with favipiravir, starting on day 0 . Despite starting ciclesonide inhalation and tocilizumab on day 2 , his $\mathrm{P} / \mathrm{F}$ ratio was 53 . On day 5, he received PMX-DHP therapy. On day 6, his dyspnea improved, as did his P/F ratio, reaching 81 on day 8 . Finally, his clinical symptoms resolved, and he was discharged from the intensive care unit without requiring mechanical ventilation. These cases indicate that PMX-DHP therapy might be a suitable treatment option for dyspnea and deoxygenation in COVID-19 pneumonia, especially in cases where an anti-inflammatory agent, such as tocilizumab, has failed to achieve the desired effect.

Keywords: blood cytokine, hypercytokinemia, P/F ratio, respiratory failure, tocilizumab

\section{Introduction}

Various types of cytokines have been implicated in the mechanism of coronavirus disease-19 (COVID-19) aggravation. ${ }^{1}$ The removal of abnormal cytokines might be a suitable treatment option in cases of severe COVID-19. However, while anticytokine treatment with tocilizumab (interleukin (IL)-6 receptor blocker) has been used in patients with COVID-19, ${ }^{2}$ there is no established treatment that targets hypercytokinemia or cytokine storms. ${ }^{3}$

Direct hemoperfusion using polymyxin B-immobilized fiber column (PMX-DHP) is a blood purification therapy used to treat septic shock, ${ }^{4}$ and acute respiratory diseases such as rapidly progressing interstitial pneumonia ${ }^{5}$ and acute exacerbation of idiopathic pulmonary fibrosis. ${ }^{6}$ COVID-19 pneumonia can also take the form of 
interstitial changes, ${ }^{7}$ but few reports are available on the use of PMX-DHP therapy in patients with COVID-19 pneumonia.

Herein, we report two treated cases without mechanical ventilation of severe COVID-19 pneumonia with deoxygenation, treated in the intensive care unit (ICU) with PMX-DHP and reduced some blood cytokine levels.

\section{Case Presentation}

\section{Case A}

A 70-year-old man with a history of hypertension and paroxysmal atrial fibrillation presented with high fever and general fatigue 4 days before hospital admission (Figure 1A). He visited our hospital due to experiencing continued fever and general malaise with mild cough. His vital signs were as follows: heart rate (HR), 92 beats/min; respiratory rate (RR), 20 breaths/min; axillary temperature, $38.3^{\circ} \mathrm{C} ; \mathrm{SaO}_{2} 97 \%$ (room air); and blood pressure, 161/91 $\mathrm{mmHg}$. Chest computed tomography (CT) scan revealed progressive interstitial pneumonia with bilateral patchy ground-glass opacities on admission (Figure 2C). The patient was diagnosed with COVID-19 pneumonia after a positive severe acute respiratory syndrome-coronavirus (SARS-CoV)-2 real-time polymerase chain reaction (PCR) test and admitted to the general ward at our hospital.

Ciclesonide inhalation was started on day 1 of hospitalization. Due to continued fever, the patient received favipiravir on day 3 (at a dose of $3600 \mathrm{mg} /$ day on day 3 and $1600 \mathrm{mg} /$ day on days 4-16). On day 5, the patient experienced dyspnea, his $\mathrm{SpO}_{2}$ was $89 \%$ at room air, and his $\mathrm{PaO}_{2} / \mathrm{F}_{\mathrm{I}} \mathrm{O}_{2}(\mathrm{P} / \mathrm{F})$ ratio was 338. Oxygen was administered as was azithromycin. On day 6 , the patient's $\mathrm{SpO}_{2}$ was under $90 \%$ (reservoir mask oxygen, $8 \mathrm{~L} / \mathrm{min}$ ) with dyspnea, leading to the administration of a single dose (8 $\mathrm{mg} / \mathrm{kg})$ of tocilizumab. Blood C-reactive protein (CRP) levels decreased after tocilizumab administration. Nevertheless, the patient's symptoms did not improve, and his $\mathrm{P} / \mathrm{F}$ ratio was 92 and accompanied by dyspnea and tachypnea. The patient was admitted to the ICU on day 7 . On day 8 , the patient's $\mathrm{P} / \mathrm{F}$ ratio decreased to 73 and his RR was $27 / \mathrm{min}$. The other blood tests before PMX-DHP on day 8 are follows: white blood cell count (WBC): 6800/ $\mu \mathrm{L}$, lymphocyte: $5.6 \%$ (absolute lymphocyte count: $526 /$ $\mu \mathrm{L}$ ), troponin I: $0.03 \mathrm{ng} / \mathrm{mL}$, NT-pro BNP: $58 \mathrm{pg} / \mathrm{mL}$, ferritin: 913 ng/mL, LDH $604 \mathrm{U} / \mathrm{L}$, AST $44 \mathrm{U} / \mathrm{L}$, procalcitonin $0.20 \mathrm{ng} / \mathrm{mL}$. The patient then received the initial round PMX-DHP therapy for $6 \mathrm{~h}$, with continued monitoring of blood cytokine levels. However, his condition did not improve, with the $\mathrm{P} / \mathrm{F}$ ratio of 89 and $\mathrm{RR}$ of $25 / \mathrm{min}$ on day 10 . He received the second round of PMXDHP therapy over $6 \mathrm{~h}$. The following day (on day 11, 10 $\mathrm{h}$ after the second round of PMX-DHP), the patient's dyspnea improved, and his RR decreased to $22 / \mathrm{min}$. On day 14 , his $\mathrm{P} / \mathrm{F}$ ratio was 149 and $\mathrm{RR}$ was 19/min. On day 18 , both his $\mathrm{P} / \mathrm{F}$ ratio and $\mathrm{RR}$ values reached 310 and $17 / \mathrm{min}$, respectively, and oxygen therapy was discontinued. On day 20, the patient was discharged from the ICU. There was no decrease in blood pressure during the course of the ICU and hemodynamics were stable On day 95, his lungs recovered without the evidence of lung fibrosis on a chest CT scan (Figure 2D). The patient recovered without requiring mechanical ventilation during his hospitalization.

\section{Case B}

A 70-year-old man with a history of hypertension, diabetes, dyslipidemia, and cerebral infarction presented with high fever 4 days before hospitalization (Figure 1B). He visited our hospital due to fever and dyspnea that he developed on the day of admission; his other symptoms were general malaise, cough, diarrhea, and disorder of smell and taste. His vital signs were as follows: HR, 62 beats/min; RR, 28 breaths/min; axillary temperature, $37.4^{\circ}$ C; $\mathrm{SaO}_{2}$ 96\% (room air); and blood pressure, 159/76 mmHg. A chest CT scan revealed progressive interstitial pneumonia with bilateral patchy ground-glass opacities on admission (Figure 2G). He was diagnosed with COVID-19 pneumonia after a positive SARS-CoV-2 real-time PCR test and admitted to the general ward at our hospital. The patient's HbA1c level was $6.3 \%$ at admission.

Favipiravir was started on the day of hospitalization (3600 mg/day on day 0 and $1600 \mathrm{mg} /$ day on days 1-13). Due to fever that persisted despite oxygen therapy, the patient received a single dose $(8 \mathrm{mg} / \mathrm{kg})$ of tocilizumab and began ciclesonide inhalation on day 2. Although his CRP level decreased immediately after tocilizumab administration, clinical symptoms such as dyspnea or tachypnea did not improve. On day 3, the patient reported worsening dyspnea, with the $\mathrm{P} / \mathrm{F}$ ratio of 53 . He was admitted to the ICU, and danaparoid sodium (2500 U/day) was started. The other blood tests before PMX-DHP on day 4 are follows: WBC: 4700/ $\mu \mathrm{L}$, lymphocyte: 11.7\% (absolute lymphocyte count: $550 / \mu \mathrm{L})$, troponin I: $0.02 \mathrm{ng} / \mathrm{mL}$, NTpro BNP: 360 pg/mL, ferritin: 1475 ng/mL, LDH 908 U/L, AST $89 \mathrm{U} / \mathrm{L}$, procalcitonin $0.24 \mathrm{ng} / \mathrm{mL}$. On day 5 , despite 

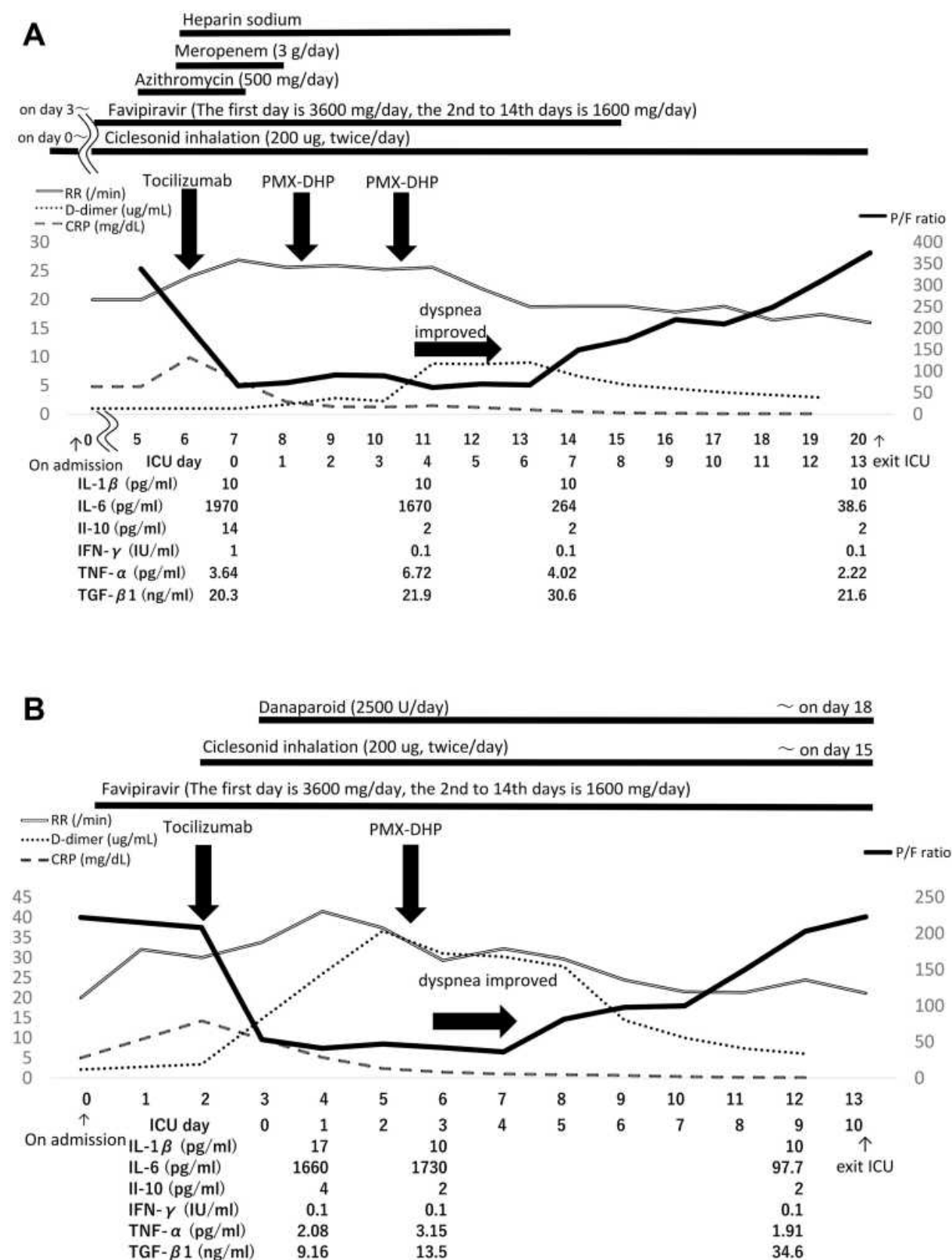

Figure I (A) Timeline of case A progression, starting on the day of admission (days). After the administration of polymyxin B-immobilized fiber column-direct hemoperfusion (PMX-DHP) therapy twice on day 10, the patient's dyspnea and respiratory rate improved on day II. On day I3, the patient's P/F ratio improved. (B) Timeline of case B progression, starting on the day of admission (days). After the administration of polymyxin B-immobilized fiber column-direct hemoperfusion (PMX-DHP) therapy on day 5 , the patient's dyspnea and respiratory rate improved on day 6 . On day 8 , the patient's P/F ratio improved. Reference values: CRP is under $0.20 \mathrm{mg} / \mathrm{dL}$, D-dimer is under $1.0 \mu \mathrm{g} / \mathrm{mL}$.

Abbreviations: CRP, C-reactive protein; $\mathrm{F}_{1} \mathrm{O}_{2}$, fraction of inspired oxygen; ICU, intensive care unit; IFN- $\gamma$, interferon-gamma; IL-I $\beta$, interleukin-I lbeta; IL-6, interleukin-6; ILI0, interleukin-10; $\mathrm{PaO}_{2}$, partial pressure of arterial oxygen; $\mathrm{PMX}-\mathrm{DHP}$, direct hemoperfusion using polymyxin $\mathrm{B}$-immobilized fiber column; $\mathrm{P} / \mathrm{F}$ ratio, $\mathrm{PaO} \mathrm{O}_{2} / \mathrm{F}_{1} \mathrm{O}_{2}$ ratio; $\mathrm{RR}$, respiratory ratio; TGF- $\beta$ I, transforming growth factor-beta I; TNF- $\alpha$, tumor necrosis factor-alpha.

tocilizumab administration, the patient's $\mathrm{P} / \mathrm{F}$ ratio was 47 and RR was 37/min; he received PMX-DHP for $6 \mathrm{~h}$, with continued monitoring of blood cytokine levels. The following day (on day $6,9 \mathrm{~h}$ after PMX-DHP administration), the patient's dyspnea improved. On day 8 , his $\mathrm{P} / \mathrm{F}$ ratio gradually improved to 81 . On day 13 , the $\mathrm{P} / \mathrm{F}$ ratio was 223 and RR was $21 /$ min. A chest X-ray confirmed improvement in aeration (Figure $2 \mathrm{~F}$ ), and the patient was discharged from the ICU. There was no decrease in blood pressure during the course of the ICU and hemodynamics were stable The patient recovered without requiring mechanical ventilation during his hospitalization. 


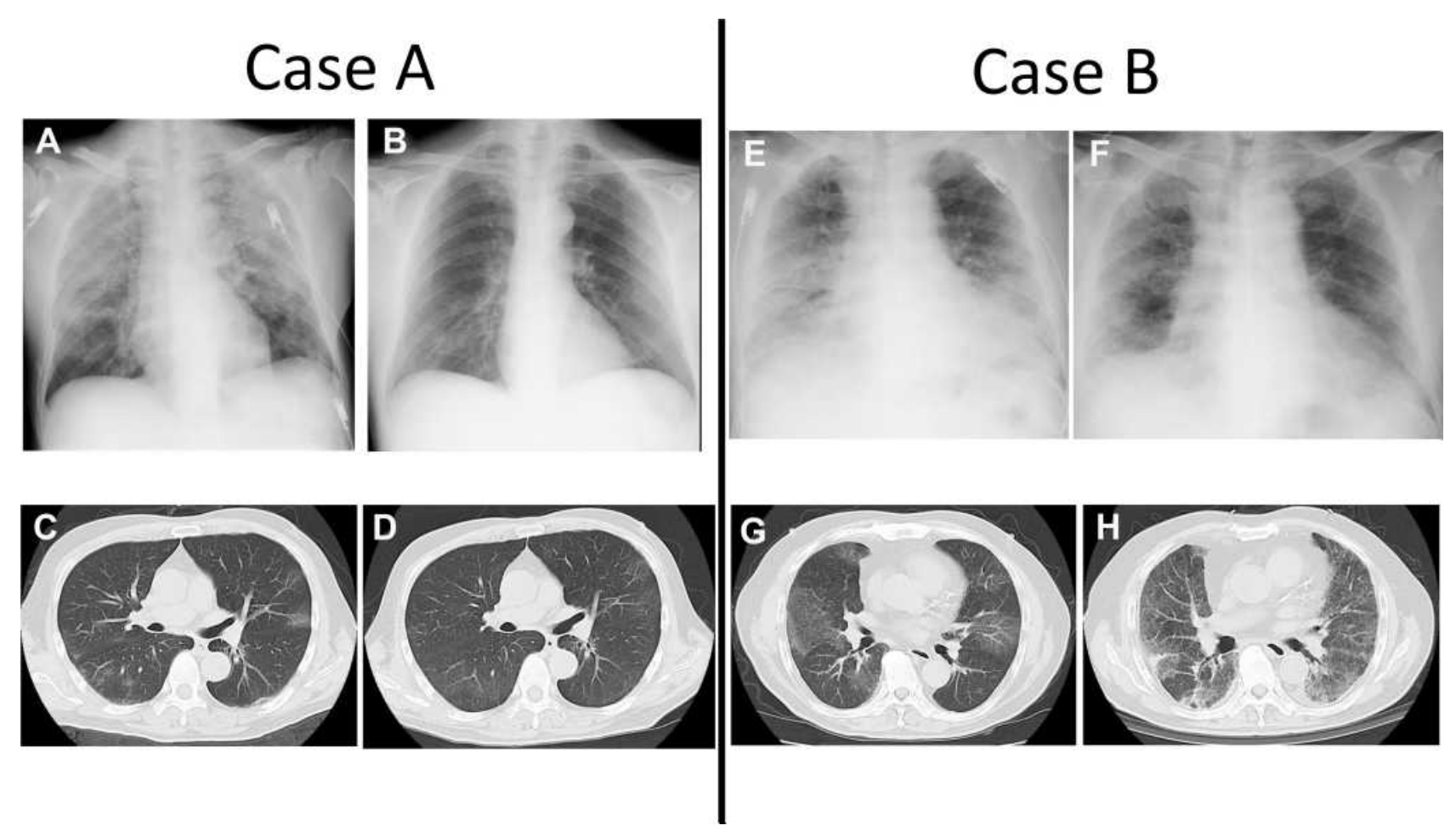

Figure 2 Serial chest X-ray and CT scans of cases (A and B). (A) CXR on day 7 (ICU day 0) shows worsening bilateral opacities. (B) CXR on day 88 shows normal lung parenchyma. (C) Chest CT scan on day 0 shows bilateral light GGOs. (D) Chest CT on day 95 shows disappearing bilateral GGOs, without the evidence of lung fibrosis. (E) CXR on day 3 (ICU day 0 ) shows the worsening of bilateral opacities. (F) CXR on day 13 shows improvement in aeration. (G) Chest CT scan on admission (day 0 ) shows light bilateral GGOs. (H) Chest CT scan on day 23 shows bilateral GGOs.

Abbreviations: CT, computed tomography; CXR, chest radiography; GGOs, ground-glass opacities.

Blood samples were obtained from both patients for cytokine level measurement before and after PMX-DHP therapy and at arbitrary clinical time points. The blood cytokines of interest were IL-1beta, IL-6, IL-10, interferon-gamma (IFN- $\gamma$ ), Tumor Necrosis Factor-alpha (TNF- $\alpha$ ) and Transforming Growth Factor (TGF)-beta 1. Serial chest X-rays and CT scans of cases A and B are presented in Figure 2.

The ethical approval requirement was waived by the Ethics Committee of Nihon University Itabashi Hospital due to the retrospective nature of this case series, which involved procedures that are part of routine care. Written consent to publish the case details was obtained from each patient.

\section{Discussion}

In both cases, these symptoms improved after treatment with PMX-DHP therapy administered over a similar period. In both patients, favorable outcomes were achieved without the use of mechanical ventilation.

The use of PMX-DHP could improve oxygenation in patients with acute respiratory distress syndrome, ${ }^{4}$ such as rapidly progressing interstitial pneumonia, ${ }^{5}$ and could inhibit inflammatory reactions in viral pneumonia. ${ }^{8}$ In fact, improvements in symptoms such as dyspnea and tachypnea were observed in the present cases. Although neither patient had data available on their blood IL-6 levels before the administration of tocilizumab, the level of IL-6 decreased after PMX-DHP therapy from 1670 to $264 \mathrm{pg} / \mathrm{mL}$ and from 1730 to $97.7 \mathrm{pg} / \mathrm{mL}$ in case A and B, respectively. Although tocilizumab has been shown in some studies to reduce mortality risk in patients with severe COVID-19, ${ }^{9,10}$ in other studies, its effects were less clear. ${ }^{11,12}$ However, in the present study, both patients improved after PMX-DHP therapy, although the mechanism through which this was achieved remains unclear. However, the reason why patients who did not respond to tocilizumab responded well to PMX-DHP is that PMXDHP has been suggested to act by reducing the levels of several inflammatory cytokines in addition to IL- $6 .{ }^{8,13}$ The evidence from the present cases indicates that the control of inflammatory responses might be an important strategy in the management of COVID-19. In fact, PMX-DHP therapy for COVID-19 with severe respiratory failure 
might help improve dyspnea and deoxygenation, leading to favorable outcomes in the present cases.

PMX-DHP therapy in the treatment of acute exacerbation of idiopathic pulmonary fibrosis significantly decreased the levels of IL-9, IL-10, IL-12, IL-17A, profibrotic cytokines platelet-derived growth factor, and vascular endothelial growth factor. ${ }^{14}$ Cytokine storm syndromes are associated with clinical severity in COVID-19 patients and have been considered therapeutic targets in the treatment of this disease; the suppression of various cytokines may improve the outcomes of patients with severe COVID-19. ${ }^{1}$ It has been suggested that tocilizumab may improve the outcomes of COVID-19 patients by blocking the IL-6 receptor. ${ }^{2,9}$ In fact, the CRP level decreased after tocilizumab administration in both of the present cases; however, clinical symptoms did not improve at the same time. In the present cases, a single dose of tocilizumab may have failed to affect the course of COVID-19 pneumonia, as the patients' levels of IL-6 were higher than those previously reported in a similar context. ${ }^{15,16}$ Moreover, in previous studies, patients with higher levels of IL-6 were more likely than their counterparts to have poor outcomes. ${ }^{17,18}$ Anti-inflammatory treatment targeting specific cytokines might affect only some of the clinical symptoms in COVID-19. Since the effectiveness of a single dose of tocilizumab in the treatment of COVID19 patients remains unclear, further research is required to determine whether a different dosing schedule, for example, a repeat dose or varied frequency of administration may be a suitable approach to patients with higher levels of IL-6. A recent report demonstrated that a combination of tocilizumab and steroid therapy for patients with COVID-19 and cytokine storms improved their clinical presentation and reduced mortality risk. ${ }^{19}$ However, the use of PMX-DHP therapy for patients with severe COVID-19 should be considered, especially, in cases where the use of anti-inflammatory agents does not achieve the desired effect. The present case series suggests that PMX-DHP therapy may reduce the level of various cytokines and improve outcomes of patients with severe respiratory failure.

\section{Conclusion}

PMX-DHP therapy might be a suitable treatment option for dyspnea and deoxygenation in patients with COVID19 pneumonia, especially in cases where tocilizumab fails to achieve the desired effect. Further research is required to establish whether PMX-DHP therapy combined with a single dose of tocilizumab can improve the outcomes of patients with COVID-19.

\section{Disclosure}

The authors report no conflicts of interest in this work.

\section{References}

1. Mehta P, McAuley DF, Brown M, et al. COVID-19: consider cytokine storm syndromes and immunosuppression. Lancet. 2020;395 (10229):1033-1034. doi:10.1016/S0140-6736(20)30628-0

2. Antwi-Amoabeng D, Kanji Z, Ford B, Beutler BD, Riddle MS, Siddiqui S. Clinical outcomes in COVID-19 patients treated with tocilizumab: an individual patient data systematic review. $J$ Med Virol. 2020;92(11):2516-2522. doi:10.1002/jmv.26038

3. Bhaskar S, Sinha A, Banach M, et al. Cytokine storm in COVID-19immunopathological mechanisms, clinical considerations, and therapeutic approaches: the REPROGRAM Consortium position paper. Front Immunol. 2020;11:1648. doi:10.3389/fimmu.2020.01648

4. Shimizu T, Miyake T, Kitamura N, Tani M, Endo Y. Endotoxin adsorption: direct hemoperfusion with the polymyxin B-immobilized fiber column (PMX). Transfus Apher Sci. 2017;56 (5):682-688. doi:10.1016/j.transci.2017.08.015

5. Ichiyasu H, Horio Y, Masunaga A, et al. Efficacy of direct hemoperfusion using polymyxin B-immobilized fiber column (PMX-DHP) in rapidly progressive interstitial pneumonias: results of a historical control study and a review of previous studies. Ther Adv Respir Dis. 2017;11(7):261-275. doi:10.1177/1753465817708950

6. Enomoto N, Mikamo M, Oyama Y, et al. Treatment of acute exacerbation of idiopathic pulmonary fibrosis with direct hemoperfusion using a polymyxin B-immobilized fiber column improves survival. BMC Pulm Med. 2015;15(1):15. doi:10.1186/s12890-015-0004-4

7. Altmayer S, Zanon M, Pacini GS, et al. Comparison of the computed tomography findings in COVID-19 and other viral pneumonia in immunocompetent adults: a systematic review and meta-analysis. Eur Radiol. 2020:1-12.

8. Takeda S, Munakata R, Abe S, et al. Hypercytokinemia with 2009 pandemic H1N1 (pH1N1) influenza successfully treated with polymyxin B-immobilized fiber column hemoperfusion. Intensive Care Med. 2010;36(5):906-907. doi:10.1007/s00134-010-1830-8

9. Xu X, Han M, Li T, et al. Effective treatment of severe COVID-19 patients with tocilizumab. Proc Natl Acad Sci U S A. 2020;117 (20):10970-10975. doi:10.1073/pnas.2005615117

10. Toniati P, Piva S, Cattalini M, et al. Tocilizumab for the treatment of severe COVID-19 pneumonia with hyperinflammatory syndrome and acute respiratory failure: a single center study of 100 patients in Brescia, Italy. Autoimmun Rev. 2020;19(7):102568. doi:10.1016/j. autrev.2020.102568

11. Furlow B. COVACTA trial raises questions about tocilizumab's benefit in COVID-19. Lancet Rheumatol. 2020;2(10):e592. doi:10.1016/ S2665-9913(20)30313-1

12. Kulanthaivel S, Kaliberdenko VB, Balasundaram K, Shterenshis MV, Scarpellini E, Abenavoli L. Tocilizumab in Sars-cov-2 patients with the syndrome of cytokine storm: a narrative review. Rev Recent Clin Trials. 2020;15. doi:10.2174/1574887115666200917110954

13. Abe S, Hayashi H, Seo Y, et al. Reduction in serum high mobility group box-1 level by polymyxin B-immobilized fiber column in patients with idiopathic pulmonary fibrosis with acute exacerbation. Blood Purif. 2011;32(4):310-316. doi:10.1159/000330325

14. Oishi K, Mimura-Kimura Y, Miyasho T, et al. Association between cytokine removal by polymyxin $\mathrm{B}$ hemoperfusion and improved pulmonary oxygenation in patients with acute exacerbation of idiopathic pulmonary fibrosis. Cytokine. 2013;61(1):84-89. doi:10.1016/ j.cyto.2012.08.032 
15. Luo P, Liu Y, Qiu L, Liu X, Liu D, Li J. Tocilizumab treatment in COVID-19: a single center experience. J Med Virol. 2020;92 (7):814-818. doi:10.1002/jmv.25801

16. Stone JH, Frigault MJ, Serling-Boyd NJ, et al. Efficacy of tocilizumab in patients hospitalized with Covid-19. N Engl J Med. 2020;383 (24):2333-2344. doi:10.1056/NEJMoa2028836

17. Chen X, Zhao B, Qu Y, et al. Detectable serum SARS-CoV-2 viral load (RNAaemia) is closely correlated with drastically elevated interleukin 6 (IL-6) level in critically ill COVID-19 patients. Clin Infect Dis. 2020;71(8):1937-1942. doi:10.1093/cid/ciaa449
18. Lin A, He Z-B, Zhang S, Zhang J-G, Zhang X, Yan W-H. Early risk factors for the duration of SARS-CoV-2 viral positivity in COVID-19 patients. Clin Infect Dis. 2020;71(16):2061-2065. doi:10.1093/cid/ ciaa490

19. Ramiro S, Mostard RLM, Magro-Checa C, et al. Historically controlled comparison of glucocorticoids with or without tocilizumab versus supportive care only in patients with COVID-19-associated cytokine storm syndrome: results of the CHIC study. Ann Rheum Dis. 2020;79(9):1143-1151. doi:10.1136/annrheumdis-2020-218479

\section{Publish your work in this journal}

Infection and Drug Resistance is an international, peer-reviewed openaccess journal that focuses on the optimal treatment of infection (bacterial, fungal and viral) and the development and institution of preventive strategies to minimize the development and spread of resistance. The journal is specifically concerned with the epidemiology of antibiotic resistance and the mechanisms of resistance development and diffusion in both hospitals and the community. The manuscript management system is completely online and includes a very quick and fair peerreview system, which is all easy to use. Visit http://www.dovepress.com/ testimonials.php to read real quotes from published authors. 